\title{
Unified dark fluid in Brans-Dicke theory
}

\author{
Sunil K. Tripathy ${ }^{1, \mathrm{a}}$, Dipanjali Behera ${ }^{2, \mathrm{~b}}$, Bivudutta Mishra ${ }^{3, \mathrm{c}}$ \\ ${ }^{1}$ Department of Physics, Indira Gandhi Institute of Technology, Sarang, Dhenkanal 759146, Odisha, India \\ 2 Department of Physics, Government College of Engineering, Kalahandi, Bandopala, Risigaon, Bhawanipatna, Kalahandi 766002, Odisha, India \\ ${ }^{3}$ Department of Mathematics, Birla Institute of Technology and Science-Pilani, Hyderabad Campus, Hyderabad 500078, India
}

Received: 5 February 2015 / Accepted: 24 March 2015 / Published online: 9 April 2015

(c) The Author(s) 2015. This article is published with open access at Springerlink.com

\begin{abstract}
Anisotropic dark energy cosmological models are constructed in the frame work of generalised BransDicke theory with a self-interacting potential. A unified dark fluid characterised by a linear equation of state is considered as the source of dark energy. The shear scalar is considered to be proportional to the expansion scalar simulating an anisotropic relationship among the directional expansion rates. The dynamics of the universe in the presence of a unified dark fluid in anisotropic background have been discussed. The presence of an evolving scalar field makes it possible to get an accelerating phase of expansion even for a linear relationship among the directional Hubble rates. It is found that the anisotropy in expansion rates does not affect the scalar field, the self-interacting potential, but it controls the non-evolving part of the Brans-Dicke parameter.
\end{abstract}

\section{Introduction}

Recent observations from distant type Ia supernovae (SNIa) suggest that currently the universe is undergoing a state of acceleration [1-5]. This intriguing discovery has led to the idea of an exotic form of energy, dubbed dark energy, that is responsible for the possible cosmic acceleration at late times. Observations of large scale structure and the cosmic microwave background (CMB) also provide strong evidence in favour of dark energy $[6,7]$. The presence of dark energy with a negative pressure is confirmed with additional evidence from observations of X-ray clusters [8], Baryon Acoustic Oscillations (BAO) [9], weak lensing [10] and integrated Sache-Wolfe effect $[11,12]$. In recent work by Sullivan et al. [13] and Suzuki et al. [14] cosmic acceleration with dark energy components has gained much support and a tighter constraint has been put on the dark energy equation of state.

\footnotetext{
a e-mail: sktripathy@igitsarang.ac.in; tripathy_sunil@ rediffmail.com

b e-mail: dipadolly@ rediffmail.com

c e-mail: bivudutta@yahoo.com
}

The exact nature of dark energy is not yet known except the fact that dark energy violates the strong energy condition and clusters only at largest accessible scales. Dark energy constitutes the highest contribution to the energy density $(68.3 \%$ dark energy, $26.8 \%$ dark matter and $4.9 \%$ baryonic matter [15-17]). A simple candidate for dark energy can be a cosmological constant in the classical FRW model with an equation of state equal to -1 . However, the cosmological constant is entangled with serious puzzles like the fine tuning problem and coincidence problem. The fine tuning problem is concerned with the theoretically predicted value of the cosmological constant from quantum field theory which is larger than the observed value by an order of $10^{123}$. Further it leads to the coincidence problem: why are we accelerating in the current epoch now that the vacuum and dust energy density are of the same order? Therefore a good number of alternative candidates have been proposed in recent times. Some alternative candidates for dark energy models are quintessence models [18], phantom models [19], ghost condensate [20] or kessence [21], holographic dark energy [22], agegraphic dark energy $[23,24]$, quintom $[25,26]$ and so on. The dark energy provides a negative pressure that generates an anti-gravity effect driving the acceleration. High resolution CMB radiation anisotropy data from Wilkinson Microwave Anisotropy Probe (WMAP) are in good agreement with the prediction of the $\Lambda$ dominated cold dark matter model $(\Lambda \mathrm{CDM})$ based upon the spatial isotropy and flatness of the universe [27], [28]. However, $\Lambda \mathrm{CDM}$ encounters some anomalous features at large scale. Even though the large scale anomalies in CMB anisotropy are still debatable, WMAP data suggest an asymmetric expansion with one direction expanding differently form the other two transverse directions at the equatorial plane [29] and signal a non-trivial topology of the large scale geometry of the universe [30,31].

The issue of global anisotropy of the universe can be simply dealt with a simple modification of the FRW model. Recently, some plane symmetric Bianchi-I models or locally 
rotationally symmetric Bianchi-I (LRSBI) models have been proposed to address the issues related to the smallness in the angular power spectrum of the temperature anisotropy [3235]. For a planar symmetry, the universe looks the same from all the points but the points all have a preferred axis. Recent Planck data shows that the primordial power spectrum of curvature perturbation is slightly redshifted from the exact scale invariance [15]. It is obvious from the Planck data that despite the notable success of $\Lambda \mathrm{CDM}$ model at high multipoles, it does not provide a good fit to the temperature power spectrum at low multipoles [15]. However, it may be noted here that there still persists uncertainty on these large angle anisotropies and they remain as open problems. LRSBI models are more general than the usual FRW models and are based on exact solutions to the Einstein field equations with homogeneous but anisotropic flat spatial sections. LRSBI models have also been studied widely, in recent times, in different contexts [36-41].

Brans-Dicke theory is a simple modification of Einstein general relativity where the purely metric coupling of matter with gravity is preserved, thus the universality of free fall (equivalence principle) is ensured [42]. Here, the gravitational constant is replaced with the inverse of a timedependent scalar field, namely, $\phi(t)=1 / 8 \pi G$, and this scalar field couples to gravity with a coupling constant $\omega$. It passes the experimental tests from the solar system [43] and is able to provide an explanation of the accelerated expansion of the universe [44]. The theory can also be tested by the observational data coming from CMB and large scale structure [45-48]. Moreover, Brans-Dicke theory arises naturally as the low energy limit of many quantum gravity theories like superstring theory or Kaluza-Klein theory. Since the Brans-Dicke theory has proved to be a better alternative to general relativity and has a dynamical framework, it evokes wide interests in the modern cosmology. In view of this, it is worthwhile to discuss dark energy models in this framework.

In the present work, we have constructed some cosmological models for LRSBI universe in the frame work of Brans-Dicke theory with a self-interacting potential and a dynamical Brans-Dicke parameter. The unified dark fluid (UDF), characterised by a linear equation of state, is considered as the source of dark energy. The paper is organised as follows: In Sect. 2, the basic equations for LRSBI universe are derived. The dynamics of evolution with a unified dark fluid characterised by a linear equation of state is discussed in Sect. 3. We have shown that a constant deceleration parameter leads to a power law for the Brans-Dicke scalar field. Also, in the work, we concentrate upon late time dynamics of the universe with an accelerated phase of expansion. At late times, the deceleration parameter is believed to be slowly varying or constant. On the other hand, a constant deceleration parameter simulates two kinds of volumetric expansion, namely: an exponential law and a power law. Cosmological models for exponential expansion and power law expansion are constructed in Sects. 4 and 5, respectively. The dynamics of universe in the presence of a dark fluid is investigated for respective models. The dynamical Brans-Dicke parameters and self-interacting potential for both models are discussed. Finally, we summarise our results in Sect. 6.

\section{Basic Equations}

We consider here the generalised Brans-Dicke theory with a self-interacting potential. In this generalised Brans-Dicke theory, the Brans-Dicke parameter is considered as a function of the scalar field $\phi$. The action for generalised Brans-Dicke theory in a Jordan frame is given by $[49,50]$

$S=\int \mathrm{d}^{4} x \sqrt{-g}\left[\phi R-\frac{\omega(\phi)}{\phi} \phi^{, \alpha} \phi_{, \alpha}-V(\phi)+L_{m}\right]$,

where $\omega(\phi)$ is the modified Brans-Dicke parameter, $V(\phi)$ is the self-interacting potential, $R$ is the scalar curvature and $L_{m}$ is the matter Lagrangian. The unit system we choose here is $8 \pi G_{0}=c=1$. Varying the action in (1) with respect to the metric tensor $g_{i j}$ and the scalar field $\phi$, the field equations are obtained as

$$
\begin{aligned}
& G_{i j}=\frac{\omega(\phi)}{\phi^{2}}\left[\phi_{i} \phi_{j}-\frac{1}{2} g_{i j} \phi_{, \alpha} \phi^{, \alpha}\right]+\frac{1}{2}\left[\phi_{, i ; j}-g_{i j} \square \phi\right], \\
& \square \phi=\frac{T}{2 \omega(\phi)+3}-\frac{2 V(\phi)-\phi \frac{\partial V(\phi)}{\partial \phi}}{2 \omega(\phi)+3}-\frac{\frac{\partial \omega(\phi)}{\partial \phi} \phi_{, i} \phi^{i}}{2 \omega(\phi)+3} .
\end{aligned}
$$

In the above equations, $T=g^{i j} T_{i j}$ is the trace of the energy momentum tensor $T_{i j}, \square$ is the d'Alembert operator. Solarsystem experiments predicted a value of the coupling constant of $\omega>40,000$ [43]. $\omega$ can be less than 40,000 on a cosmological scale [45]. Observational constraints on the Brans-Dicke model were obtained in a flat universe with cosmological constant and cold dark matter using the latest WMAP and SDSS data [47]. Within the $2 \sigma$ range, the value of $\omega$ satisfies $\omega<-120.0$ or $\omega>97.8$. In a recent work, the Brans-Dicke parameter is constrained from the combination of observational data of CMB from seven year WMAP, BAO from SDSS, SNIa data from union2 and the $\mathrm{X}$-ray gas mass fraction data from Chandra X-ray observations of the largest relaxed galaxy clusters to be in the range $0.0014<\frac{1}{\omega}<0.0024$ or $417<\omega<714$ [51]. The rate of change of $G$ was constrained to be $-1.7510^{-12}$ year $^{-1}<$ $\frac{\dot{G}}{G}<1.0510^{-12}$ year $^{-1}$ at $2 \sigma$ confidence level in the present epoch [47]. Brans-Dicke theory reduces to Einstein's general relativity in the limit of a constant scalar field and an infinitely large Brans-Dicke parameter $\omega$. However, this consideration may not hold always good $[41,52,53]$. 
A plane symmetric LRSBI model is considered through the metric

$\mathrm{d} s^{2}=-\mathrm{d} t^{2}+A^{2} \mathrm{~d} x^{2}+B^{2}\left(\mathrm{~d} y^{2}+\mathrm{d} z^{2}\right)$,

where $A$ and $B$ are the directional scale factors and are considered as functions of cosmic time only. The metric corresponds to considering the $y z$-plane as the symmetry plane and $x$ as the axis of symmetry. The eccentricity of such a universe is given by $e=\sqrt{1-A^{2} / B^{2}}$. The expansion scalar $\theta$ for this metric is $\theta=\frac{\dot{A}}{A}+2 \frac{\dot{B}}{B}$, where an overhead dot represents an ordinary time derivative. Defining the directional Hubble parameters along the axis of symmetry and symmetry plane as $H_{1}=\frac{\dot{A}}{A}$ and $H_{2}=\frac{\dot{B}}{B}$, the mean Hubble parameter can be written as $H=\frac{1}{3}\left(H_{1}+2 H_{2}\right)$ and $\theta=3 H$. The scalar expansion can be expressed in terms of the directional Hubble parameters as

$\theta=H_{1}+2 H_{2}$.

The shear scalar for the plane symmetric metric defined in (4) is expressed as

$\sigma^{2}=\frac{1}{2}\left[\Sigma_{i} H_{i}^{2}-\frac{1}{3} \theta^{2}\right]=\frac{1}{3}\left(H_{1}-H_{2}\right)^{2}$.

The shear scalar may be taken to be proportional to the expansion scalar which envisages a linear relationship between the directional Hubble parameters $H_{1}$ and $H_{2}$ as $H_{1}=k H_{2}$. This assumption leads to an anisotropic relation between the directional scale factors $A$ and $B$ as $A=B^{k}$. Here, $k$ is an arbitrary positive constant that takes care of the anisotropic nature of the model. If $k=1$, the model reduces to be isotropic and otherwise the model is anisotropic. One may note that such an assumption is not new and is widely used in the literature to handle anisotropic models. The mean Hubble parameter can now be expressed as $H=\frac{1}{3}(k+2) H_{2}$. The average anisotropic parameter $\mathcal{A}=\frac{1}{3} \Sigma\left(\frac{\Delta H_{i}}{H}\right)^{2}$ for the model is $\mathcal{A}=2\left(\frac{k-1}{k+2}\right)^{2}$. Obviously for an isotropic model with $k=1, \mathcal{A}$ vanishes and has a finite non-zero value for anisotropic models. One should keep in mind that the universe is observed to be mostly isotropic and any deviation from isotropic behaviour must be considered as a sort of small perturbation.

The field equations, for a cosmic fluid with energy momentum tensor $T_{i j}=(\rho+p) u_{i} u_{j}+p g_{i j}$, now assume the explicit forms

$$
\begin{aligned}
& 9(2 k+1) H^{2}=(k+2)^{2} \\
& \times\left[\frac{\rho}{\phi}+\frac{\omega(\phi)}{2}\left(\frac{\dot{\phi}}{\phi}\right)^{2}-3 H\left(\frac{\dot{\phi}}{\phi}\right)+\frac{V(\phi)}{2 \phi}\right],
\end{aligned}
$$

$$
\begin{aligned}
& 6(k+2) \dot{H}+27 H^{2}=(k+2)^{2} \\
& \quad \times\left[-\frac{p}{\phi}-\frac{\omega(\phi)}{2}\left(\frac{\dot{\phi}}{\phi}\right)^{2}-\frac{6 H}{(k+2)}\left(\frac{\dot{\phi}}{\phi}\right)-\frac{\ddot{\phi}}{\phi}+\frac{V(\phi)}{2 \phi}\right], \\
& 3\left(k^{2}+3 k+2\right) \dot{H}+9\left(k^{2}+k+1\right) H^{2}=(k+2)^{2} \\
& \quad \times\left[-\frac{p}{\phi}-\frac{\omega(\phi)}{2}\left(\frac{\dot{\phi}}{\phi}\right)^{2}-\frac{3(k+1) H}{(k+2)}\left(\frac{\dot{\phi}}{\phi}\right)-\frac{\ddot{\phi}}{\phi}+\frac{V(\phi)}{2 \phi}\right],
\end{aligned}
$$

and the Klein-Gordon wave equation for the scalar field,

$$
\frac{\ddot{\phi}}{\phi}+3 H \frac{\dot{\phi}}{\phi}=\frac{\rho-3 p}{2 \omega(\phi)+3}-\frac{\frac{\partial \omega(\phi)}{\partial \phi} \dot{\phi}^{2}}{2 \omega(\phi)+3}-\frac{2 V(\phi)-\phi \frac{\partial V(\phi)}{\partial \phi}}{2 \omega(\phi)+3}
$$

where $\rho$ is the dark energy density and $p$ is the dark energy pressure.

Subtracting Eq. (9) from Eq. (8), we can obtain the evolution equation for the Brans-Dicke scalar field,

$-\frac{\dot{H}}{H}-3 H=\frac{\dot{\phi}}{\phi}$,

which can also be expressed as

$(q-2) H=\frac{\dot{\phi}}{\phi}$,

where $q=-1-\frac{\dot{H}}{H^{2}}$ is the deceleration parameter. It should be mentioned here that a positive deceleration parameter describes a decelerating universe whereas a negative $q$ implies an accelerating one. Equation (12) implies that, for a non-static universe $(H \neq 0)$, a constant scalar field will give us a decelerating universe with $q=2$. Brans-Dicke field equations with constant scalar field reduce to the usual Einstein field equations in general relativity. Therefore, one can conclude that in general relativity accelerating models cannot be achieved for LRSBI models by assuming a linear relationship among the directional Hubble rates. This issue has already been investigated earlier [38,54] and similar results have been obtained. However, in the present work, it is interesting to note that the presence of an evolving Brans-Dicke field modifies the situation and it is possible to get accelerating models even if the directional Hubble rates are proportional to each other. Again, the behaviour of the Brans-Dicke field is governed by the deceleration parameter and the consequent Hubble rate. For a constant deceleration parameter the Brans-Dicke field evolves as $\phi \sim a^{q-2}$, or more specifically $\phi \sim(1+z)^{2-q}$, where $a$ is the scale factor, related to the redshift $z$ by $\frac{1}{a}=1+z$. Here, we consider the scale factor at the present epoch to be 1 . In other words, a constant deceleration parameter favours a power law for the BransDicke scalar field. Moreover, it has become a usual practice, in the literature, to use a power law scalar field $\left(\phi=\phi_{0} a^{\alpha}\right)$ to address different issues in cosmology in the framework of 
Brans-Dicke theory. Also one should keep in mind that Eq. (12) is valid only for an anisotropic model with $k \neq 1$.

The general expressions for the Brans-Dicke parameter and the self-interacting potential can be obtained from the field equations (7)-(9) as

$$
\begin{aligned}
\omega(\phi)= & \left(\frac{\dot{\phi}}{\phi}\right)^{-2}\left[-\frac{\rho+p}{\phi}-\frac{\ddot{\phi}}{\phi}+\frac{3 k H}{k+2} \frac{\dot{\phi}}{\phi}\right. \\
& \left.-\frac{6 \dot{H}}{k+2}-\frac{18(1-k)}{(k+2)^{2}} H^{2}\right], \\
V(\phi)= & 2 \phi\left[\frac{9(2 k+1) H^{2}}{(k+2)^{2}}-\frac{\rho}{\phi}-\frac{\omega(\phi)}{2}\left(\frac{\dot{\phi}}{\phi}\right)^{2}+3 H \frac{\dot{\phi}}{\phi}\right] .
\end{aligned}
$$

The behaviour of the Brans-Dicke parameter and the selfinteracting potential along with the dynamics of the universe can be understood if we know the behaviour of the energy density, pressure and the scale factor of the universe. The scale factor of the universe can be fixed from the behaviour of the deceleration parameter or the assumed dynamics of the late time accelerated universe. For the pressure and energy density, usually, a barotropic relationship in the form $P=P(\rho)$, known as the equation of state, is assumed. In this sense many equations of state with different mathematical formulations have been proposed in the literature to address different issues in cosmology. In the present work, we assume a linear equation of state to handle the issue of the dark energy problem in the frame work of generalised Brans-Dicke theory.

\section{Unified dark fluid}

A dark fluid model with a linear equation of state was proposed in the spirit of the generalised Chaplygin gas model (GCM) $[55,56]$ after its success in addressing issues related to the late time cosmic acceleration and dark energy problem. Also CGM is known to be quite consistent with observations [57]. Holeman and Naidu in their work in Ref. [56] coined the linear equation of state defining the dark fluid as wet dark fluid (WDF), claiming that such an equation of state was used earlier to treat water and an aqueous solution $[58,59]$. In UDF, a constant adiabatic sound speed is assumed and the equation of state is obtained through an integration over the energy density. The integration constant coming out in the process, obviously, has a behaviour similar to the cosmological constant and the equation of state has components both from the dark matter and the dark energy sectors. This is usually referred to as dark degeneracy.
A unified fluid dark energy is modelled through the equation of state

$p=\gamma\left(\rho-\rho^{*}\right)$,

where $\gamma$ and $\rho^{*}$ are positive constants. This non-homogeneous linear equation of state (15) provides a description of both hydro-dynamically stable $(\gamma>0)$ and unstable $(\gamma<0)$ fluids [55]. One may notice here that the UDF equation of state contains two parts - one behaves as the usual barotropic cosmic fluid, and the other behaves as a cosmological constant and unifies the dark energy and dark matter components. The adiabatic speed of sound for this equation of state is $C_{s}^{2}=\gamma$. For stability of a model the adiabatic speed of sound should be $C_{s}^{2} \geq 0$ and for causality, $C_{s}^{2} \leq 1$. Hence, $\gamma$ should lie in the range of $0 \leq \gamma \leq 1 . \gamma=0$ refers to the case of dark matter and $\gamma=1$ implies a stiff fluid dominated with dark energy (maybe the contribution coming from other sources such as a fluid with a bulk viscosity or a cosmological constant). The value of $\gamma$ in between zero and 1 refers to an exotic cosmic fluid unifying both the dark energy and the dark matter and it deals with the dark sector of the universe. However, there are no such constraints for $\rho^{*}$ and it can be treated as a free parameter. The advantage of the equation of state (15) is that dark energy can be described with a positive squared sound speed (contrary to the need of a negative squared sound speed in phantom energy). In Ref. [56], Holman and Naidu have claimed that the WDF model (similar to UDF) is consistent with SNIa observations [3], WMAP data $[60,61]$ and constraints coming from the measurements of the matter power spectrum [62]. They have shown that a WDF model with $\gamma=0.316228$ fits well to the observed data. Babichev et al. [55] did not put any sign constraint on the parameters $\gamma$ and $\rho^{*}$. For different combinations of these two parameters they obtained distinctive types of the cosmic evolution scenario such as Big Bang, Big Crunch, Big Rip, anti-Big Rip solutions with de Sitter attractor and bouncing solutions. They have shown that, for $1+\gamma>0$ and $\gamma \rho^{*}>0$ the universe may contain either non-phantom or phantom energy, whereas for $1+\gamma>0$ and $\gamma \rho^{*}<0$ the universe may contain only phantom energy leading to a Big Crunch. On the other hand, for $1+\gamma<0$ and $\gamma \rho^{*}<0$, the universe may contain either non-phantom or phantom energy, whereas for $1+\gamma<0$ and $\gamma \rho^{*}>0$, the universe may contain only phantom energy leading to a Big Rip in a finite time. The WDF equation of state is considered as a linearised equation of state of any smooth function $p=p(\rho)$ in the vicinity of some local point. UDF dark energy model has generated a considerable research interest in recent times and has been studied widely addressing different issues in relativity and cosmology [63-71].

The parameters of the UDF can be constrained using the observational data on the dark energy equation of state. In the present work, we use the recent observational constraint 


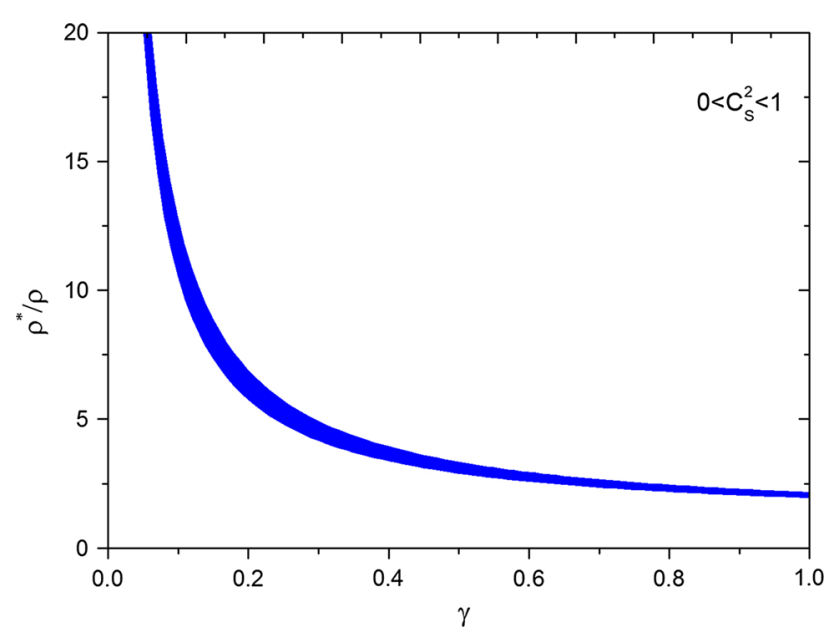

Fig. 1 Observational constraints on the UDF parameters

on the dark energy equation of state, $\omega_{D}=-1.06_{-0.13}^{+0.11}$ [72]. The range of allowed values for the parameters $\gamma$ and $\rho^{*}$ as obtained by using the data of Ref. [72] is shown in Fig. 1. In the figure, $\gamma$ is restricted within the range $0 \leq \gamma \leq 1$ based upon the stability and causality of the model which keeps the parameter $\rho^{*}$ in the positive domain for negative $\omega_{D}$. In a recent work, Liao et al. [71] have constrained the parameters of a unified dark fluid described through a two parameter affine linear equation of state similar to the one discussed in this work using the Hubble parameter data $H(z)$, type Ia Supernovae data from Union 2 datasets, Baryon Acoustics Oscillations observations from Sloan Digital Sky Survey and the CMB radiation data from WMAP. They have constrained the parameter $\gamma$ to be $0.00172_{-0.00479}^{+0.00392}$ in $1 \sigma$ for a flat universe and $0.00242_{-0.00775}^{+0.0078}$ in $1 \sigma$ for a non-flat universe. In another work, Xu et al. [69] constrained this parameter to be $0.000487_{-0.000487}^{+0.000117}$ in $1 \sigma$ confidence. So far, it is believed that a low value of $\gamma$ much less than 1 fits the observational data well.

The energy conservation equation for the matter field is given by

$\dot{\rho}+3 H(\rho+p)=0$.

For the unified dark fluid equation of state, (16) can be integrated to get

$\rho=\rho_{\Lambda}+\rho_{\gamma} a^{-3(1+\gamma)}$

where $\rho_{\Lambda}=\frac{\gamma \rho^{*}}{1+\gamma}$ and $\rho_{\gamma}=\left(\rho_{0}-\rho_{\Lambda}\right) \cdot a=\left(A B^{2}\right)^{\frac{1}{3}}$ is the average radius scale factor of the universe. $\rho_{0}$ is the dark energy density at the present epoch. Since $\gamma$ and $\rho^{*}$ are positive, $\rho_{\Lambda}$ is positive, varying between 0 and $\frac{\rho^{*}}{2}$ for $\gamma=0$, $\gamma=1$, respectively. Depending upon the relative values of $\rho_{0}$ and $\rho_{\Lambda}, \rho_{\gamma}$ can either be positive or negative. It is interesting to note that the dark energy density has two parts:

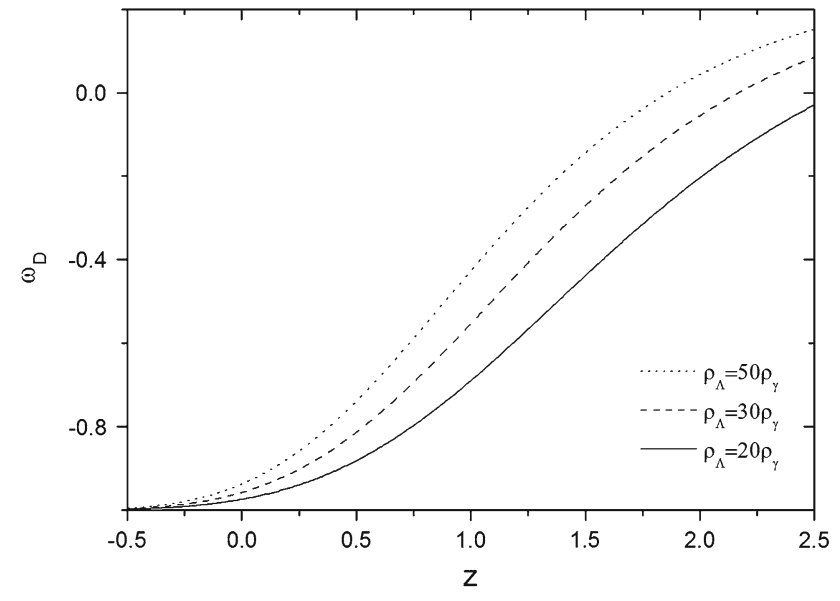

Fig. 2 Dark energy equation of state as a function of redshift for three positive values of the ratio $\frac{\rho_{\Lambda}}{\rho_{\gamma}} \cdot \gamma$ is taken to be 0.316

one behaves like a cosmological constant and the other part dynamically evolves with the cosmic expansion.

The dark energy pressure can be expressed as

$p=-\rho_{\Lambda}+\gamma \rho_{\gamma} a^{-3(1+\gamma)}$,

so that the equation of state parameter $\omega_{D}=\frac{p}{\rho}$ becomes

$\omega_{D}=-1+\frac{1+\gamma}{1+\left(\frac{\rho_{\Lambda}}{\rho_{\gamma}}\right) a^{3(1+\gamma)}}$.

The dynamical evolution of the dark energy equation of state can also be assessed from

$\omega_{D}=-1+\frac{1+\gamma}{1+\left(\frac{\rho_{\Lambda}}{\rho_{\gamma}}\right)(1+z)^{-3(1+\gamma)}}$.

The dark energy pressure and the dark energy equation of state parameter also have two parts each, one corresponds to the usual cosmological constant and the second part evolves dynamically with cosmic expansion. In Fig. 2, the dynamical evolution of the dark energy equation of state parameter is shown as a function of redshift for three representative values of the ratio $\frac{\rho_{\Lambda}}{\rho_{\gamma}}=20,30$ and 50 corresponding to $\omega_{D}=-0.937,-0.958$ and -0.974 at the present epoch. $\gamma$ is chosen to be 0.316 . $\omega_{D}$ dynamically evolves from $\gamma$ at an early epoch to -1 at late times of evolution. In the intermediate time zone, the behaviour of the dark energy equation of state is the same for all the choices of $\frac{\rho_{\Lambda}}{\rho_{\gamma}}$, except the fact that with increase in the value of the ratio, $\omega_{D}$ becomes less negative. In Fig. 3 , the dark energy equation of state is plotted as a function of redshift with $\gamma=0.316$ for three negative values of the ratio $\frac{\rho_{\Lambda}}{\rho_{\gamma}}=-8,-20$ and -50 , corresponding to $\omega_{D}=-1.19,-1.07$ and -1.03 at the present epoch. The dark energy equation of state evolves in the phantom region and increases with the cosmic expansion to behave like a cosmological constant. 


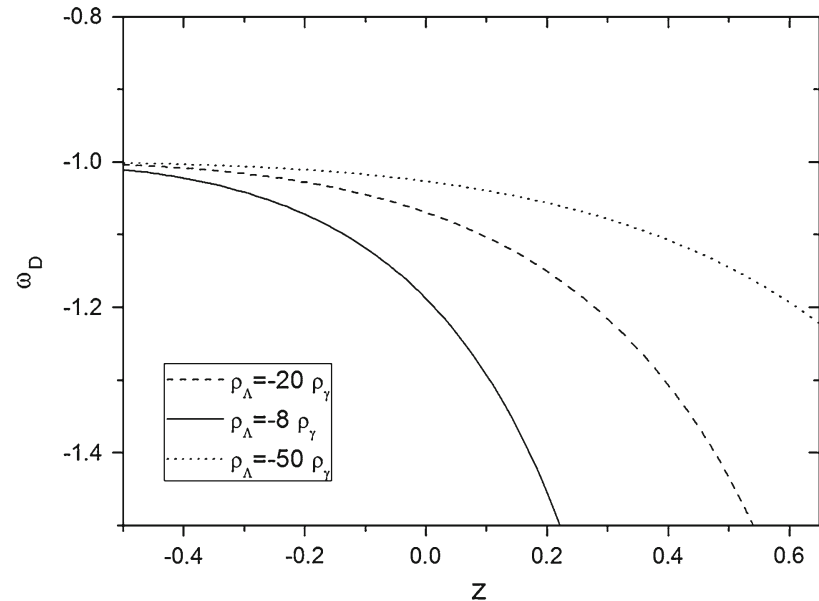

Fig. 3 Dark energy equation of state as a function of redshift for three negative values of the ratio $\frac{\rho_{\Lambda}}{\rho_{\gamma}} \cdot \gamma$ is taken to be 0.316

Deceleration parameter $q=-\frac{\ddot{a}}{a H^{2}}$ and jerk parameter $j=\frac{\dddot{a}}{a H^{3}}$ are considered as important quantities in the description of the dynamics of universe. The observational constraints as set upon these parameters in the present epoch from type Ia supernova and X-ray cluster gas mass fraction measurements are $q_{0}=-0.81 \pm 0.14$ and $j_{0}=2.16 \pm_{-0.76}^{+0.81}$ [73]. In a recent work, the deceleration parameter is constrained from $H(z)$ and SNIa data to be $q=-0.34 \pm 0.05$ [74]. Experimentally it is challenging to measure the deceleration parameter and jerk parameter and one needs to observe objects of red shift $z \geq 1$. In attempts to investigate the accelerated expansion of the universe, the sign and behaviour of these parameters have been considered in different manners in different works. The time variation of the deceleration parameter is under debate even though, in certain models, a time varying $q$ leads to a cosmic transit from early deceleration to late time acceleration [75-78]. However, at a late of time of cosmic expansion, the deceleration parameter is believed to vary slowly with time or to become a constant. A constant deceleration parameter leads to two different volumetric expansions of the universe, namely the power law expansion and exponential expansion. In a model with exponential expansion, the radius scale factor increases exponentially with time, leading to a constant Hubble rate. In a model with power law expansion of the volume scale factor, the scale factor can be expressed as a cosmic time raised to some positive power. The Hubble parameter for such a power law model behaves reciprocally to the cosmic time. In the present work, we are interested in models describing a late time universe with the predicted cosmic acceleration and therefore we will consider the exponential and power law expansion of the scale factor corresponding to a constant and variable (decaying) mean Hubble rate, i.e. $H=H_{0}$ and $H=\frac{m}{t}$, where $H_{0}$ and $m$ are positive constants. It is worth to mention here that

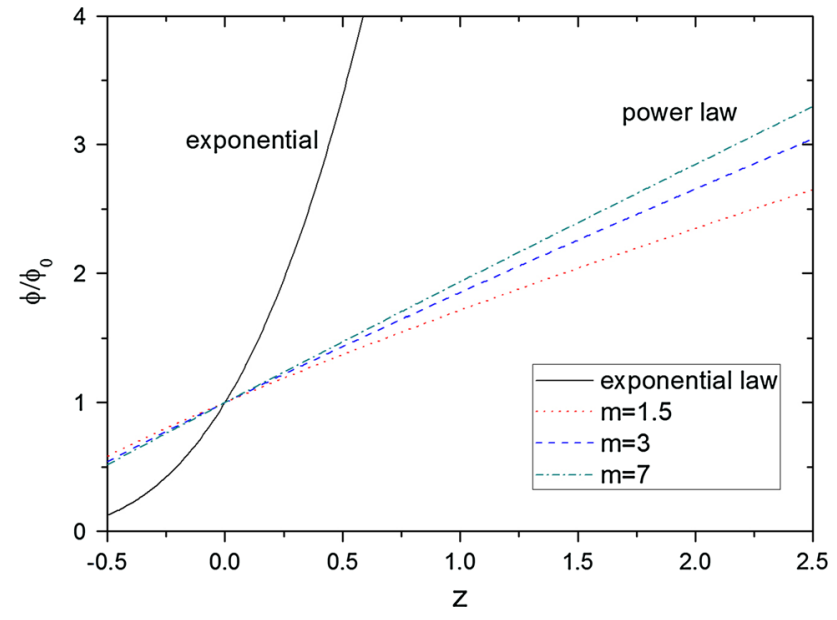

Fig. 4 Evolution of Brans-Dicke scalar field. Brans-Dicke fields for both the exponential and the power law models are shown. For the power law model, three representative values of the exponent $m$ are considered

the choice of a constant deceleration parameter cannot provide a time dependent cosmic transition from a deceleration phase in the past to an accelerated phase at late times.

\section{Exponential model}

In this kind of volumetric expansion, the Hubble rate is a constant quantity i.e. $H=H_{0}=$ constant and the scale factor is given by $a=e^{H_{0}\left(t-t_{0}\right)}$ and it describes a de Sitter type universe. $t_{0}$ is the cosmic time in the present epoch. The directional scale factors along the longitudinal and transverse directions are $A=e^{\frac{3 k H_{0}\left(t-t_{0}\right)}{(k+2)}}$ and $B=e^{\frac{3 H_{0}\left(t-t_{0}\right)}{(k+2)}}$. The deceleration parameter and jerk parameter, for this choice of the Hubble rate, are $q=-1$ and $j=1$. The directional deceleration parameters $q_{x}, q_{y}$ and $q_{z}$ are the same as that of the mean deceleration parameter $q$.

Integration of (12) yields for an exponential scale factor

$\phi=\phi_{0} e^{-3 H_{0}\left(t-t_{0}\right)}$,

where $\phi_{0}$ is the value of the scalar field in the present epoch. In terms of the scale factor and redshift $z$, we can express the scalar field, respectively, as $\phi=\phi_{0} a^{-3}$ and $\phi=\phi_{0}(1+z)^{3}$, where we have used the fact $\frac{1}{a}=1+z$. In Fig. 4 , the evolution of the Brans-Dicke scalar field is plotted as a function of redshift. The scalar field decreases exponentially from a large value at the early epoch to vanishing value at late times of the cosmic evolution.

The rest energy density and pressure for the present model are

$\rho=\rho_{\Lambda}+\rho_{\gamma}\left(\frac{\phi}{\phi_{0}}\right)^{1+\gamma}$, 
$p=-\rho_{\Lambda}+\gamma \rho_{\gamma}\left(\frac{\phi}{\phi_{0}}\right)^{1+\gamma}$.

The rest energy density and pressure in the model evolve with the scalar field. They decrease from higher values in the past to low values in a later period. At late times, $\rho$ dynamically evolves to become $\rho_{\Lambda}$ and the pressure $p$ reduces to $-\rho_{\Lambda}$. At late times, a negative pressure dominates the scenario and helps in the acceleration of cosmic expansion.

Using the fact that $\frac{\dot{\phi}}{\phi}=-3 H_{0}$ and $\frac{\ddot{\phi}}{\phi}=9 H_{0}^{2}$ we get the Brans-Dicke parameter as

$\omega(\phi)=\omega_{0}+\omega_{1} \phi^{\gamma}$

where $\omega_{0}=-2\left[\frac{\left(k^{2}+2 k+3\right)}{(k+2)^{2}}\right]$ and $\omega_{1}=-\left[\frac{(\gamma+1) \rho_{\gamma}}{9 H_{0}^{2}}\right] \phi_{0}^{-(1+\gamma)}$. It is interesting to note here that the Brans-Dicke parameter has two parts: a constant $\omega_{0}$ and a dynamically evolving part. The constant part is decided from the anisotropic nature of the model. For an isotropic model with $k=1$, it becomes $\omega_{0}=-\frac{4}{3}$. The anisotropic nature of the model does not affect the evolving part of the Brans-Dicke parameter. The evolving part is mostly governed by the value of $\gamma$. The variable Brans-Dicke parameter becomes a constant for the lower limit of $\gamma$, whereas it varies linearly with the scalar field for its upper limit. The allowed range of the Brans-Dicke parameter is $\omega_{0}+\omega_{1} \leq \omega(\phi) \leq \omega_{0}+\omega_{1} \phi$. The role played by the parameter $\rho^{*}$ is quite interesting. In the absence of this parameter, the cosmic fluid behaves as a barotropic fluid with the usual relation $p=\gamma \rho$, and $\omega_{1}$ turns out to be negative. Consequently, the Brans-Dicke parameter assumes a much higher negative value in the early phase of cosmic evolution. However, in the presence of this parameter, the value of $\omega(\phi)$ is a bit lifted up because of the positive contribution from $\rho^{*}$. For the particular choice of $\rho^{*}=\left(1+\frac{1}{\gamma}\right) \rho_{0}, \omega_{1}$ vanishes and $\omega(\phi)$ behaves as a constant $\omega_{0}$. In Fig. 5, the functional $\omega_{B D}=\frac{\omega(\phi)-\omega_{0}}{\omega_{1}}$ is shown as a function of the scalar field for the exponential scale factor leading to a de Sitter kind of universe. The shaded area in the plot shows the allowed range of the functional $\omega_{B D}$ corresponding to the upper and lower bounds on $\gamma$. The blue curve running through the shaded area is for the representative value $\gamma=0.316$. It is obvious from the figure that, for this representative value of $\gamma$, the functional $\omega_{B D}$ increases with increase in the scalar field. At an early phase of the cosmic evolution, the functional is almost constant or has a little variation with the scalar field, whereas, with the growth of time, the rate of change in the functional becomes more rapid at late times. It can be concluded that with the cosmic expansion, the functional $\omega_{B D}$ decreases for $\gamma>0$. The rate of decrement slows down as the value of $\gamma$ decreases from its upper bound to the lower one. For $\gamma=0$, the functional becomes a constant with a value equal to 1 . However, for $\gamma=1$, the value of $\omega$ is decided by the param-

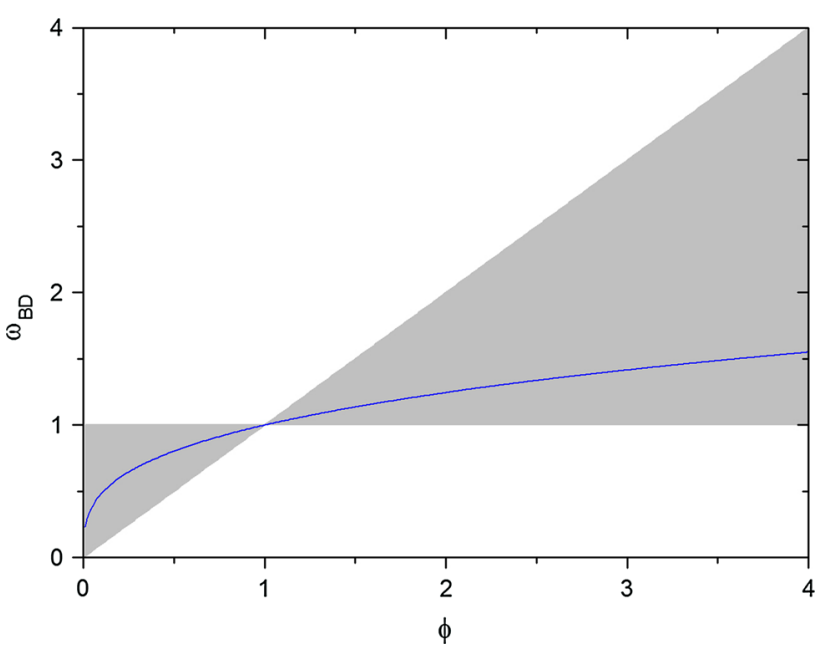

Fig. 5 The functional $\omega_{B D}$, for the exponential model, as a function of scalar field. The shaded area shows the allowed range for the functional. The curve running through the shaded area is for $\gamma=0.316$

eters $\rho^{*}, \rho_{0}, \phi_{0}$ and $H_{0}$. The scalar field decreases with time and therefore, for any value of $\gamma$ other than zero, the BransDicke parameter evolves to a constant $\omega_{0}$ at late times of the evolution. From a dimensional consistency as demanded by the Klein-Gordon wave equation (10), for $\gamma \neq 0$, the value of $\omega_{0}$ should be -1.5 , which favours the anisotropic parameter $k$ to be 4 . On the other hand, the average anisotropic parameter is constrained from WMAP data [79] to be $|\sqrt{\mathcal{A}}|=10^{-5}$, which corresponds to $k=1.0000212$ in our present model. In fact, the universe is observed to be mostly flat and isotropic and hence the anisotropy in cosmic expansion must be considered as a little perturbation to the isotropic behaviour.

The self-interacting potential can be expressed as

$V(\phi)=V_{0}+V_{1} \phi^{1+\gamma}$

where $V_{0}=-2 \rho_{\Lambda}$ and $V_{1}=-2 \rho_{\gamma} \phi_{0}^{-(1+\gamma)}$. The selfinteracting potential does not depend upon the anisotropic parameter $k$, rather it depends upon the parameters of the unified dark fluid. For the lower limit of $\gamma$, the self-interacting potential varies linearly with the scalar field and for the upper limit it varies in a quadratic manner. For a particular choice of the parameter $\rho^{*}=\left(1+\frac{1}{\gamma}\right) \rho_{0}$, the Brans-Dicke parameter behaves like a constant with values $\omega_{0}=-1.5$ and the selfinteracting potential behaves as a constant with the value of $V(\phi)=V_{0}=-2 \rho_{0}$. With the evolution of the scalar field, the self-interacting potential evolves to a constant value of $-2 \rho_{\Lambda}$ at late times. However, in the absence of the parameter $\rho^{*}$ in the dark energy equation of state, the potential vanishes. In other words, the presence of the parameter $\rho^{*}$ induces a self-interacting potential even in the absence of a scalar field. The behaviour of the functional $V_{B D}=\frac{V-V_{0}}{V_{1}}$ is shown in Fig. 6. The shaded area in the graph shows the allowed range 


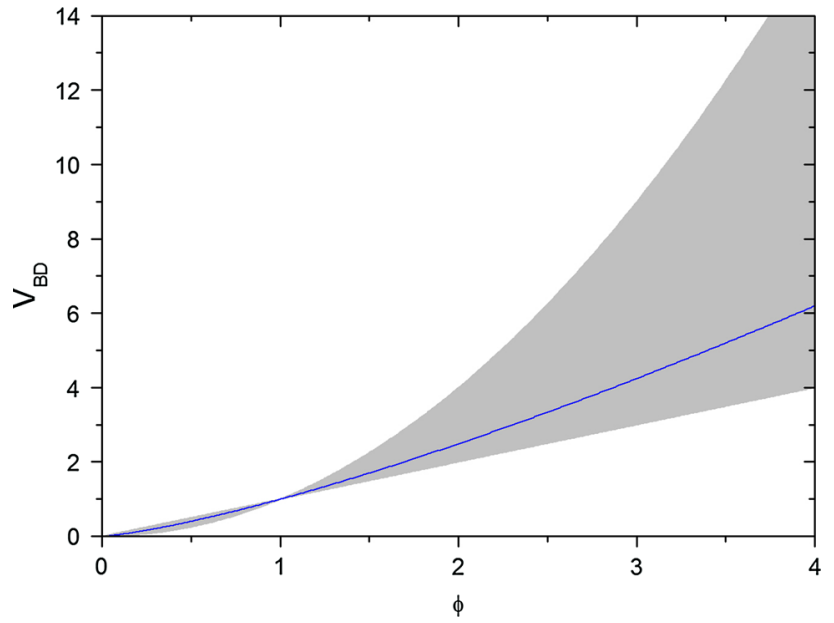

Fig. 6 The functional $V_{B D}$, for the exponential model, as a function of scalar field. The shaded area shows the allowed range for the functional. The curve running through the shaded area is for $\gamma=0.316$

of the functional $V_{B D}$. The curve running through the shaded area is for $\gamma=0.316$, where the functional $V_{B D}$ increases with the increase in the scalar field. The slope of the curve increases with the increase in $\gamma$.

The dynamics of cosmic evolution through its expansion history can be understood from the dark energy equation of state parameter, $\omega_{D}$. From (22) and (23), we get

$\omega_{D}=-1+\frac{1+\gamma}{1+\left(\frac{\rho_{\Lambda}}{\rho_{\gamma}}\right)\left(\frac{\phi}{\phi_{0}}\right)^{-(1+\gamma)}}$.

The dark energy equation of state does not depend on the anisotropic nature of the model and depends on the parameters of the UDF like the self-interacting potential. The dark energy equation of state, for $\gamma>0$, decreases from $\gamma$ in the quintessence region at the initial epoch to behave as a cosmological constant with $\omega_{D}=-1$, at a later epoch when the scalar field vanishes. At a given cosmic time, the dark energy equation of state is decided by the parameters $\gamma$ and $\rho^{*}$. One should note the role played by the parameter $\rho^{*}$. In the absence of this parameter, i.e. for $\rho_{\Lambda}=0$, the dark energy equation of state is simply given by $\omega_{D}=\gamma$, which can take only positive values as decided from the constraints on the adiabatic speed of sound. But the inclusion of $\rho^{*}$ into the equation of state modifies the relation and makes the dark energy equation of state a dynamic one. In other words, $\rho^{*}$ incorporates some negative pressure simulating the dark energy necessary for the accelerated expansion.

The time variation of Newtonian gravitational constant is given by

$\frac{\dot{G}}{G}=\frac{\dot{\phi}}{\phi}=-3 H_{0}$.
Since, in the present model, the Hubble parameter is assumed to be a constant quantity throughout the cosmic evolution, obviously, $\frac{\dot{G}}{G}$ turns out to be a constant and its value can be calculated in a straightforward manner. The observational data from $H(z)$ and Supernovae Ia constrained the Hubble parameter as $H_{0}=68.93_{-0.52}^{0.53} \mathrm{kms}^{-1} \mathrm{Mpc}^{-1}$ [74] and accordingly the time variation of $G$ can be calculated from the present model.

\section{Power law model}

In the case of power law expansion with the Hubble parameter behaving as $H=\frac{m}{t}, m$ being a positive constant, the average scale factor behaves as $a=\left(\frac{t}{t_{0}}\right)^{m}$. The scale factors along the longitudinal and transverse directions read $A=\left(\frac{t}{t_{0}}\right)^{\left(\frac{3 m k}{k+2}\right)}$ and $B=\left(\frac{t}{t_{0}}\right)^{\left(\frac{3 m}{k+2}\right)} \cdot$ Cosmologies with a power law scale factor are widely discussed in the literature [74,80-86]. The success of the power law model lies with the fact that models with $m \geq 1$ do not encounter the horizon problem and do not witness the flatness problem. In Ref. [74], from the analysis of observational constraints from $H(z)$ and SNIa data, Kumar has shown that a power law cosmology is viable in the description of the acceleration of the present day universe even though it fails to produce primordial nucleosynthesis.

The deceleration parameter for this model is $q=\frac{1}{m}-1$. In order to be in the safe zone for accelerated expansion, the predicted deceleration parameter should be negative and that can be achieved only if $m>1$. In terms of the deceleration parameter, the parameter $m$ can be expressed as $m=\frac{1}{1+q}$. Considering the observational constraints from Ref. [73], we put the constraints on $m$ to be $3.03 \leq m \leq 20$. Corresponding to the constraints from Ref. [74], $m$ can be constrained in the range $1.4085 \leq m \leq 1.6393$. The jerk parameter is calculated to be $j=\frac{(m-1)(m-2)}{m}$ and can be constrained in the range $0.69 \leq j \leq 17.1$ [73] and $-0.1716 \leq j \leq-0.1407$ [74]. It is worth to mention here that the exact determination of the jerk parameter involves the observation of high-z supernovae, which is a tough task. Therefore, current observational data have not yet been able to pin down the range or sign of the jerk parameter. The directional Hubble rates for this model are $H_{1}=\left(\frac{3 m k}{k+2}\right) \frac{1}{t}$ and $H_{2}=\left(\frac{3 m}{k+2}\right) \frac{1}{t}$. Consequently the directional deceleration parameters along different spatial directions are obtained using the relation $q_{i}=-1+\frac{d}{d t}\left(\frac{1}{H_{i}}\right)$ as $q_{x}=\frac{k+2}{3 m k}-1$ and $q_{y}=q_{z}=\frac{k+2}{3 m}-1$. The mean deceleration parameter $q$ is obtained from the directional deceleration parameters as $q=\frac{1}{3}\left(q_{x}+q_{y}+q_{z}\right)$. The directional deceleration parameters are also independent of time. For an isotropic model, $k=1$ and the directional 
deceleration parameters all reduce to $q_{x}=q_{y}=q_{z}=\frac{1}{m}-1$ and become equal to the mean $q$.

The scalar field for this model becomes

$\phi=\phi_{0}\left(\frac{t}{t_{0}}\right)^{1-3 m}$.

In terms of the scale factor $\phi=\phi_{0}(a)^{\frac{1-3 m}{m}}$ and in terms of the redshift $\phi=\phi_{0}(1+z)^{\frac{3 m-1}{3 m}}$. It is obvious from (28) that the scalar field decreases with expansion of the universe and vanishes at large cosmic time. The behaviour of the scalar field is only decided by the single parameter $m$ or more specifically the constant negative deceleration parameter. The scalar field is independent of the anisotropic parameter $k$. In Fig. 4, the scalar field for the model is shown as a function of redshift. In the figure we have considered three representative values of the exponent $m$, namely $1.5,3$ and 7 , which are within the allowed range as calculated from the observational data for the deceleration parameter. It is amply clear from the figure that a model with a higher value of $m$ has a higher scalar field in the past, whereas it has a low value of the scalar field in the future. Also, the variation of scalar field with $m$ at early time is much better exemplified than that at late times of the evolution.

The energy density and pressure for this model with power law expansion read

$\rho=\rho_{\Lambda}+\rho_{\gamma}\left(\frac{\phi}{\phi_{0}}\right)^{\frac{3 m(1+\gamma)}{3 m-1}}$

and

$p=-\rho_{\Lambda}+\gamma \rho_{\gamma}\left(\frac{\phi}{\phi_{0}}\right)^{\frac{3 m(1+\gamma)}{3 m-1}}$.

Just like the previous model, the energy density and pressure evolve with the scalar field from large values at the initial epoch to, respectively, become $\rho_{\Lambda}$ and $-\rho_{\Lambda}$ at large cosmic time.

The variable Brans-Dicke parameter can be expressed as

$\omega(\phi)=\omega_{0 p}+\omega_{1 p} \phi^{\left(\frac{3 \gamma m-1}{3 m-1}\right)}$,

where $\omega_{0 p}=\frac{3 m[(k+2)(k-3 m k+2)-6 m(1-k)]}{(1-3 m)^{2}(k+2)^{2}}$ and $\omega_{1 p}=$ $-\frac{(\gamma+1) \rho_{\gamma}}{(1-3 m)^{2}} t_{0}^{2} \phi_{0}^{-\frac{3 \gamma m-2}{3 m-1}}$. We have used the fact $\frac{\dot{\phi}}{\phi}=\frac{1-3 m}{t}$ and $\frac{\ddot{\phi}}{\phi}=\frac{3 m(3 m-1)}{t^{2}}$ to get (31) from (14). It is interesting to note that the Brans-Dicke parameter is a function of the scalar field even in the lower limit of $\gamma$, in which it decreases with the scalar field. In other words, the Brans-Dicke parameter assumes lower values in the past and larger values in the late time of cosmic evolution. If we consider the upper bound of $\gamma$, the Brans-Dicke parameter evolves linearly with the scalar field. The anisotropic nature of the model affects only the constant part of the Brans-Dicke parameter. The

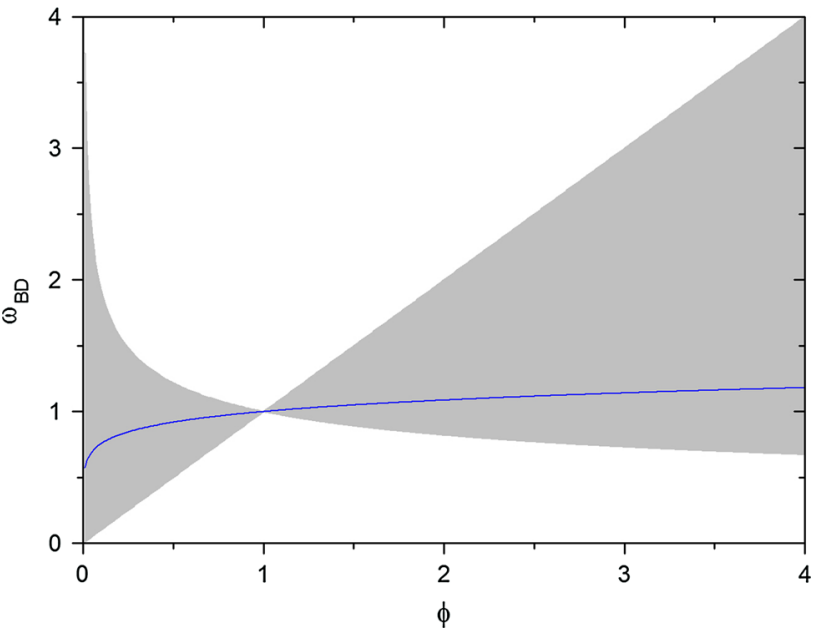

Fig. 7 The functional $\omega_{B D}$, for the power law model, as a function of scalar field. The shaded area shows the allowed range for the functional. The curve running through the shaded area is for $\gamma=0.316$

behaviour of the evolving part is governed by the parameters of the UDF and the exponent $m$. In Fig. 7, the functional $\omega_{B D}=\frac{\omega-\omega_{0} p}{\omega_{1 p}}$ is plotted as a function of the Brans-Dicke field. The shaded area shows the allowed range. In order to get a general behaviour, we have shown the functional for a representative value $\gamma=0.316$. For the upper bound of $\gamma$, the functional linearly behaves with the Brans-Dicke field. In order to calculate the lower bound for the functional we have used a reasonable value of the exponent $m=1.5$, which lies within the observational limits corresponding to more recent data. Just like the previous model, the functional $\omega_{B D}$ for the representative value of $\gamma$ varies slowly with the Brans-Dicke field at an early epoch and varies rapidly at a late time of evolution. $\rho^{*}$ has a significant role to play in the behaviour of the Brans-Dicke parameter. For the particular choice $\rho^{*}=\left(1+\frac{1}{\gamma}\right) \rho_{0}$, it behaves as a pure constant which can be equated to -1.5 , from dimensional consistency of the Klein-Gordon wave equation.

The self-interacting potential for this model is given by

$V(\phi)=V_{0}+V_{1 p} \phi^{\frac{3 m(1+\gamma)}{3 m-1}}$,

where

$V_{1 p}=(\gamma-1) \rho_{\gamma} \phi_{0}^{\left(\frac{3 m(1+\gamma)}{1-3 m}\right)}$.

Since $m>1$, the self-interacting potential increases with the increase in the scalar field. Like the previous model, the scalar field does not depend on the anisotropic exponent $k$ and it depends on the parameters of the unified dark fluid. For a choice of $\rho^{*}=\left(1+\frac{1}{\gamma}\right) \rho_{0}$ or $\gamma=1$, the selfinteracting potential becomes independent of the scalar field 


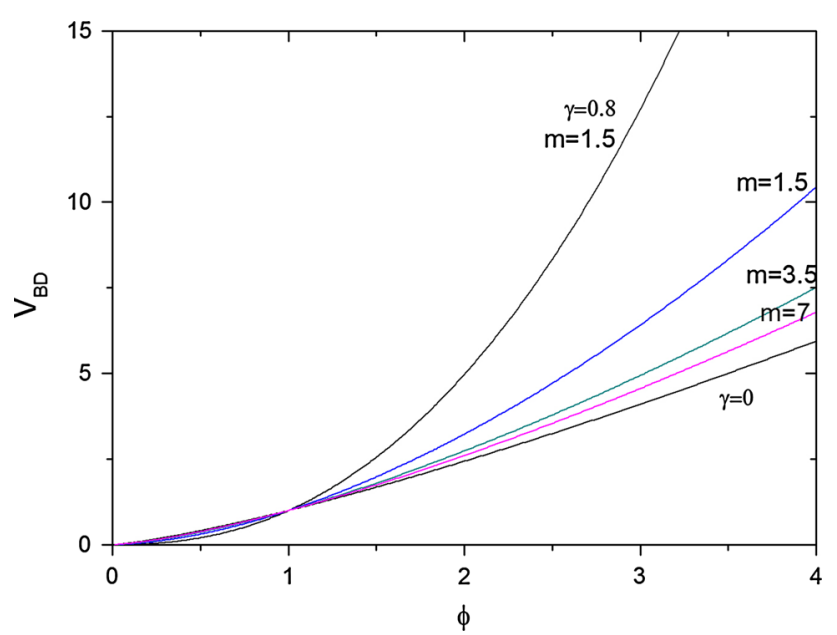

Fig. 8 The functional $V_{B D}$, for the power law model, as a function of scalar field. The upper curve is for $\gamma=0.8$ and $m=1.5$. The lower curve shows the lower bound with $\gamma=0$. The three curves in the middle are for three different values of the exponent $m$ with $\gamma=0.316$

and equals $-2 \rho_{\Lambda}$. This is the same value as the potential assumes at a later epoch. In other words, there is an induced self-interacting potential in the absence of the scalar field, because of the parameter $\rho^{*}$. In Fig. 8, we have shown the functional $V_{B D}=\frac{V-V_{0}}{V_{1 p}}$ as a function of the Brans-Dicke field. In this figure we cannot set up the upper bound since $V_{1 p}$ vanishes for $\gamma=1$. However, a curve for $\gamma=0.8$ with $m=1.5$ is shown in the figure to get an idea. The curves for $\gamma=0.316$ are shown for three different values of $m$, e.g. $m=1.5,3.5$ and 7 . The functional $V_{B D}$ decreases with the decrease in the field and at late times of the evolution it vanishes. For a given value of $\gamma$, the functional decreases with the increase in $m$ at early epochs, whereas it increases at late times. However, the rate of increment at late times is less as compared to the rate of decrement at an early phase.

The dark energy equation of state $\omega_{D}$ can be calculated from (29) and (30) as

$\omega_{D}=-1+\frac{1+\gamma}{1+\left(\frac{\rho_{\Lambda}}{\rho_{\gamma}}\right)\left(\frac{\phi}{\phi_{0}}\right)^{\frac{3 m(1+\gamma)}{1-3 m}}}$.

The dark energy equation of state decreases from $\gamma$ in the beginning to behave like a cosmological constant with $\omega_{D}=-1$ at a late epoch of cosmic evolution. In the absence of the parameter $\rho^{*}$, the dark energy equation of state is a constant quantity i.e. $\gamma$. The presence of this parameter makes the dark energy equation of state an evolving one. The anisotropic nature of the model does not affect $\omega_{D}$. However, the dark energy equation of state is controlled by the choice of the exponent $m$, which is decided by the observational constraints on the deceleration parameter and the jerk parameter.
The time variation of the Newtonian gravitational constant for this power law model is

$\frac{\dot{G}}{G}=\frac{1-3 m}{t}$.

Here, $\frac{\dot{\phi}}{\phi}=\frac{1-3 m}{t}$ inversely varies with time. The value of $m$ for the present is constrained from the observational data [74] and consequently the time variation of $G$ can be predicted to be in the range $-3.918<\frac{\dot{G}}{G} t<-3.226$.

\section{Conclusion}

In the present work, we have constructed some cosmological models mimicking the late time cosmic acceleration in the frame work of generalised Brans-Dicke scalar tensor theory of gravitation for a plane symmetric universe. The cosmic fluid is considered to be a dark fluid described by a two parameter affine equation of state. The shear scalar is considered to be proportional to the scalar expansion, which simulates a linear relationship among the directional Hubble rates incorporating anisotropy in expansion rates along different spatial directions. In general relativity, such an assumption does not provide an accelerating model. However, in the frame work of generalised Brans-Dicke theory with evolving scalar field, it is possible to get accelerated phase of expansion with such an assumption. Considering a constant deceleration parameter at a late time of evolution of the universe, we have considered two kinds of volume expansion, namely, the power law expansion and the exponential law of expansion. Moreover, we have shown that a constant deceleration parameter leads to a power law in the Brans-Dicke scalar field. The presence of the extra term in the barotropic fluid equation of state makes the dark energy equation of state an evolving one. The dark energy equation of state evolves from a positive constant quantity equal to the adiabatic speed of sound in the beginning to behave like a cosmological constant at a later epoch of cosmic evolution. The scalar field is found to decrease with the cosmic expansion. The self-interacting potential increases with the increase in scalar field. In an initial epoch, the self-interacting potential is having a large value and decreases with time to have a constant value decided by the equation of state parameter at a later epoch. The anisotropic nature of the model does not affect the behaviour of the scalar field and the self-interacting potential. However, the non-evolving part of the dynamic Brans-Dicke parameter is affected by the introduction of an anisotropy in the expansion rates.

Acknowledgments BM acknowledges University Grants Commission, New Delhi, India, for financial support to carry out the Minor Research Project [F.No-42-1001/2013(SR)]. SKT likes to thank Saha Institute of Nuclear Physics, Kolkata, India, for providing necessary facilities where a part of this work is done. 
Open Access This article is distributed under the terms of the Creative Commons Attribution 4.0 International License (http://creativecomm ons.org/licenses/by/4.0/), which permits unrestricted use, distribution, and reproduction in any medium, provided you give appropriate credit to the original author(s) and the source, provide a link to the Creative Commons license, and indicate if changes were made.

Funded by $\mathrm{SCOAP}^{3}$.

\section{References}

1. S. Perlmutter et al., Nature 391, 51 (1998)

2. A.G. Reiss et al., Astron. J. 116, 1009 (1998)

3. A.G. Reiss et al., Astron. J. 607, 665 (2004)

4. R. Knop et al., Astrophys. J. 598, 102 (2003)

5. A.G. Reiss et al., Astrophys. J. 659, 98 (2007)

6. D.N. Spergel et al., Astrophys. J. S. 170, 377 (2007)

7. A. Blanchard et al., Astrophys. J. 659, 98 (2007)

8. S.W. Allen et al., Mon. Not. R. Astron. Soc. 353, 457 (2004)

9. D.J. Eisenstein et al., Astrophys. J. 633, 560 (2005)

10. C.R. Contaldi, H. Hoekstra, A. Lewis, Phys. Rev. Lett. 90, 221303 (2004)

11. S.P. Boughn, R.G. Critendron, Nature 427, 45 (2004)

12. S. Cole et al., Mon. Not. R. Astron. Soc. 362, 505 (2005)

13. M. Sullivan et al., Astrophys. J. 737, 102 (2011)

14. N. Suzuki et al., Astrophys. J. 746, 85 (2012)

15. P.A.R. Ade et al., ( Planck Collaboration) (2013). arXiv:1303.5076 [astro-ph.CO]

16. P.A.R. Ade et al., ( Planck Collaboration) (2013). arXiv:1303.5082 [astro-ph.CO]

17. P.A.R. Ade et al., ( Planck Collaboration) (2013). arXiv:1303.5084 [astro-ph.CO]

18. B. Ratra, P.J.E. Peebles, Phys. Rev. D 37, 321 (1998)

19. R.R. Caldwell, Phys. Lett. B 545, 23 (2002)

20. F. Piazza, S. Tsujikawa, J. Cosmol. Astropart. Phys. 0407, 004 (2004)

21. T. Chiba, T. Okabe, M. Yamaguchi, Phys. Rev. D 62, 023511 (2000)

22. B. Wang, C.Y. Lin, D. Pavon, E. Abadalla, Phys. Lett. B 662, 01 (2008)

23. R.G. Cai, Phys. Lett. B 657, 228 (2007)

24. H. Wei, R.G. Cai, Phys. Lett. B 660, 113 (2008)

25. E. Elizalde, S. Nojiri, S.D. Odintsov, Phys. Rev. D 70, 043539 (2004)

26. S. Nojiri, S.D. Odintsov, S. Tsujikawa, Phys. Rev. D 71, 063004 (2005)

27. G. Hinshaw et al., WMAP Collaboration, Astrophys. J. Suppl. Ser. 180, 225 (2009)

28. M.R. Nolta et al., WMAP collaboration, Astrophys. J. Suppl. Ser. 180, 296 (2009)

29. R.V. Buiny, A. Berera, T.W. Kephart, Phys. Rev. D 73, 063529 (2006)

30. M. Watanabe, S. Kanno, J. Soda, Phys. Rev. Lett. 102, 191302 (2009)

31. A. de Oliveira-Costa, M. Tegmark, M. Zaldarriaga, A. Hamilton, Phys. Rev. D 69, 063516 (2004)

32. L. Campanelli, P. Cea, L. Tedesco, Phys. Rev. Lett. 97, 131302 (2006)

33. L. Campanelli, Phys. Rev. D 80, 063006 (2009)

34. L. Campanelli, P. Cea, L. Tedesco, Phys. Rev. D 76, 063007 (2007)

35. A. Gruppo, Phys. Rev. D 76, 083010 (2007)

36. S.K. Tripathy, S.K. Nayak, S.K. Sahu, T.R. Routray, Astrophys. Space Sci. 321, 247 (2009)

37. S.K. Tripathy, D. Behera, T.R. Routray, Astrophys. Space Sci. 325, $93(2010)$

38. S.K. Tripathy, Astrophys. Space Sci. 325, 93 (2014)
39. S.K. Tripathy, K.L. Mahanta, Eur. Phys. J. Plus 130, 30 (2015). arXiv: 1407.7792

40. T. Koivisto, D.F. Mota, J. Cosmol. Astropart. Phys. 806, 18 (2008)

41. M. Sharif, S. Waheed, Eur. Phys. J. C 72, 1876 (2012)

42. X.L. Liu, X. Zhang, Commun. Theor. Phys. 52, 761 (2009)

43. B. Bertotti, L. Iess, P. Tortora, Nature (London) 425, 374 (2003)

44. C. Mathiazhagan, V.B. Johri, Class. Quant. Grav. 1, L29 (1984)

45. V. Acquaviva, L. Verde, J. Cosmol. Astropart. Phys. 0712, 001 (2007)

46. S. Tsujikawa, K. Uddin, S. Mizuno, R. Tavakol, J. Yokoyam, Phys. Rev. D 77, 103009 (2008)

47. F. Wu, X. Chen, Phys. Rev. D 82, 083003 (2010)

48. F. Wu, X. Chen, Phys. Rev. D 88, 084053 (2013)

49. K. Nordvedt Jr, Astrophys. J. 161, 1059 (1970)

50. R.V. Wagoner, Phys. Rev. D. 1, 3209 (1970)

51. H. Alavirad, A. Sheykhi, Phys. Lett. B 734, 148 (2014)

52. C. Romero, A. Barros, Phys. Lett. A 173, 243 (1993)

53. A.K. Yadav, B. Saha, Astrophys. Space Sci. 337, 759 (2012)

54. S.K. Tripathy, Int. J. Theor. Phys. 52, 4218 (2013)

55. E. Babichev, V. Dokuchaev, Yu. Eroshenko, Class. Quant. Grav. 22, 143 (2005). arXiv:astro-ph/0407193

56. R. Holman, S. Naidu (2005). arXiv:astro-ph/0408102

57. H.B. Sandvik, M. Tegmark, M. Zaldarriaga, I. Waga, Phys. Rev. D 69, 123524 (2004)

58. P.G.Tait, The Voyage of HMS Challenger, vol. 2 (London, HMSO, 1888), pp. 1-73

59. A.T.J. Hyward, Brit. J. Appl. Phys. 18, 965 (1967)

60. C.L. Bennet et al., Astrophys. J. Supl. 148, 1 (2003)

61. D.N. Spergel et al., Astrophys. J. Supl. 148, 175 (2003)

62. M. Tegmark et al., A.J.S. Hamilton, Y. Xu.: Mon. Not. R. Astron. Soc. 335, 887 (2002)

63. R. Scherrer, Phys. Rev. D. 73, 043502 (2006)

64. B. Mishra, P.K. Sahoo, Astrophys. Space Sci. 349, 491 (2013)

65. T. Chiba, N. Sugiyama, T. Nakamura, Mon. Not. R. Astron. Soc. 289, L5 (1997)

66. T. Chiba, N. Sugiyama, T. Nakamura, Mon. Not. R. Astron. Soc. 301, 72 (1998)

67. K.N. Ananda, M. Bruni, Phys. Rev. D 74, 023523 (2006)

68. A. Balbi, M. Bruni, C. Quercellini, Phys. Rev. D 76, 103519 (2007)

69. L. Xu, Y. Wang, H. Noh, Phys. Rev. D 85, 043003 (2012)

70. W. Wang, L. Xu, Phys. Rev. D 88, 023505 (20132)

71. K. Liao, S. Cao, J. Wang, X. Gong, Z.H. Zhu, Phys. Lett. B 710, 17 (2012)

72. S. Kumar, L. Xu, Phys. Lett. B 737, 244 (2014)

73. D. Rapetti, S.W.Allen, M.A. Amin, R.D. Blandford, Mon. Not. R. Astron. Soc. 375, 1510 (2007). arXiv:astro-ph/0605683

74. S. Kumar, Mon. Not. R. Astron. Soc. 422, 2532 (2012). arXiv:1109.6924 [astro-ph]

75. A.K. Yadav, A. Sharma, Res. Astron. Astrophys. 13, 501 (2013)

76. K.S. Adhav, Eur. Phys. J. Plus 126, 122 (2011)

77. O. Akarsu, T. Dereli, Int. J. Theor. Phys. 51, 612 (2012)

78. A. Pradhan, S. Otarod, Astrophys. Space Sci. 311, 413 (2007)

79. L. Campanelli et al., Int. J. Mod. Phys. D 20, 1153 (2011)

80. S. Gehlaut, P. Kumar, G. Sethi, D. Lohiya (2003). arXiv:astro-ph/0306448

81. A. Dev, M. Safanova, D. Jain, D. Lohiya, Phys. Lett. B 548, 12 (2002)

82. G. Sethi, P. Kumar, S. Pandey, D. Lohiya, Spacetime Subs. 6, 31 (2005)

83. A. Batra, M. Sethi, D. Lohiya, Phys. Rev. D 60, 108301 (1999)

84. A. Batra, D. Lohiya, S. Mahajan, A. Mukherjee, Int. J. Mod. Phys. D 9, 757 (2000)

85. M. Kaplinghat, G. Steigman, I. Tkachev, T.P. Walker, Phys. Rev. D 59, 043514 (1999)

86. C.P. Singh, V. Singh, Gen. Relativ. Gravit. 46, 1696 (2014) 